\title{
Intra-articular injection of xanthan gum: A potential therapy for osteoarthritis
}

\author{
Huarong Shao ${ }^{1,2}$, Guanying Han ${ }^{1,2}$, Peixue Ling ${ }^{1,2^{*}}$, Xiqiang Zhu ${ }^{2}$ Fei Liu ${ }^{2}$, Yan $\mathrm{Jin}^{2}$, Lijuan Zhao, \\ Tianmin Zhang ${ }^{1}$
}

${ }^{1}$ School of Pharmaceutical Sciences, Shandong University, Jinan, China

${ }^{2}$ Post-Doctoral Scientific Research Workstation, Institute of Biopharmaceuticals of Shandong Province, Jinan, China

Email: *1px@sdfmg.com

Received 26 June 2012; revised 26 July 2012; accepted 6 August 2012

\begin{abstract}
Osteoarthritis (OA) means inflammation of the joints, with the symptoms of joint pain, stiffness, and swelling of the joints. It is a degenerative disease that appears to be caused by both biomechanical and biochemical factors. Intra-articular (IA) injection treatment is one of the main treatment methods for $\mathrm{OA}$ because of its positive effect in reducing joint pain and increasing joint mobility. IA injection of xanthan gum (XG) could protect the joint cartilage, relieve the synovitis and reduce the $O A$ progression in experiment OA. The injection of XG may have a long-lasting effect in the joint cavity, which could avoid frequent IA injections. However, for the development of this potential therapy, further studies such as the effective long-term pain relief properties, the detailed action mechanism and the pharmacokinetics of the XG injection will be conducted. This article briefly reviewed the preparation, safety evaluation, pharmacodynamics and possible action mechanism of XG injection, and come up with the ideas for further development of this potential therapy for $O A$.
\end{abstract}

Keywords: Osteoarthritis; Xanthan Gum;

Pharmacodynamics; Action Mechanism

\section{INTRODUCTION}

Osteoarthritis (OA) is the most common joint disorder and is a major cause of disability and impaired mobility worldwide [1]. It is characterized by cartilage degradation and synovial inflammation which is directly related to clinical symptoms such as joint swelling, synovitis and pain [2]. Since both aging [3] and obesity [4] are associated with increasing rates of $\mathrm{OA}$, the prevalence of $\mathrm{OA}$ is likely to continue to rise. Development of new OA treatment method is important and necessary to reduce

\footnotetext{
"Corresponding author.
}

its public health impact.

Current pharmacotherapy aims at symptom relief and cartilage protection. It includes systemic analgesics, nonsteroid antiinflammatory drugs (NSAID) and intra-articular (IA) injections with glucocorticoids and viscosupplementation agents such as sodium hyaluronate $(\mathrm{SH})[5$, 6]. IA injection of SH is widely used in the treatment of $\mathrm{OA}$, and clinical trails showed that SH therapy is effective and safe for the OA patients [7-9]. The main objective of IA injection of SH is to make up for the loss of viscoelastic properties of OA synovial fluid and to protect against degradation of cartilage [10-12]. However, SH will be quickly degraded through hydrolytic and enzymatic reactions in vivo due to its instability [13]. Therefore, a compound possessing similar function to $\mathrm{SH}$, but with a longer efficacy is really needed to avoid frequent IA injections.

Xanthan gum (XG) is a high molecular weight anionic polysaccharide produced from microbial fermentation [14]. It is similar to SH in viscosity and rheology $[15,16]$, however, it is likely to be more stable than SH and not be easily degraded in vivo [17]. Previous studies demonstrated that IA injection of XG could protect the joint cartilage, relieve the synovitis and reduce the OA progression in an experiment OA model [17]. XG injection probably has a long-lasting protective effect on articular cartilage to avoid numerous injections. In this article we briefly reviewed the preparation, safety evaluation, pharmacodynamics and possible action mechanism of XG injection, and come up with the ideas for further development of this potential therapy for OA.

\section{BRIEF INTRODUTION OF XG}

XG (Figure 1) is a microbial extracellular heteropolysaccharide produced by the bacterium Xanthomonas campestris. Its molecular weight distribution ranging from $2 \times 10^{6}$ to $2 \times 10^{7} \mathrm{Da}$ [14]. The primary structure of $\mathrm{XG}$ is based on repeated pentasaccharide units. The backbone chain consists of two glucose units linearly by 


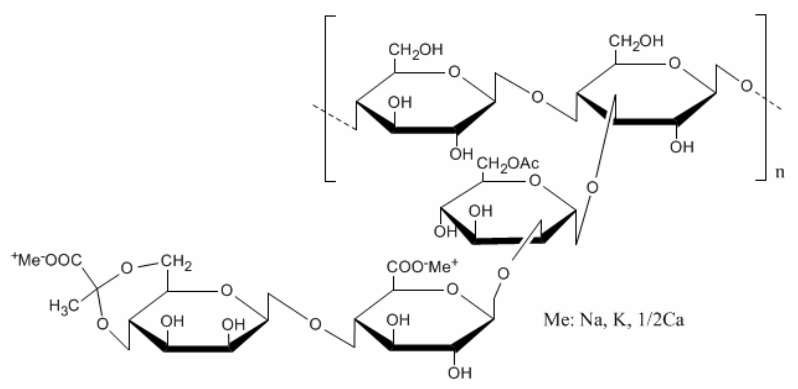

Figure 1. Xanthan gum.

$\beta 1 \rightarrow 4$ linkages, and the trisaccharide side chain is composed of two D-mannose units alternating D-glucuronic acid. The internal mannose unit is acetylated and on approximately half of the terminal D-mannose units residues a pyruvic acid moiety $[18,19]$. The secondary structure of XG is a fivefold helical structure formed by the trisaccharide side chain wrapping back the main chain through hydrogen bonds. The main chain is protected by the side chains from the outside environment. The tertiary structure of XG is a net work structure formed by helical complex [14].

The properties of $X G$ are quite different and unusual for its special structure, such as a high viscosity even at low concentrations, a high degree of pseudoplastic, stability and compatibility with most metallic salts, excellent solubility and stability in acidic and alkaline solutions and resistance to degradation at elevated temperatures and various $\mathrm{pH}$ levels [20]. Besides, XG is nontoxic and biocompatible.

XG has been approved for its use in the production of foods by the United States Food and Drug Adminstration (FDA) without any specific quantity limitations in 1969 [20]. Now, XG has been widely used in variety of foods for its special and important properties. XG is also well known to be employed as a thickener in many other industries such as papermaking, textile and cosmetics industries [14]. XG is biocompatible allowing its use in various medical and pharmaceutical applications such as topical ocular application [21,22], implantation [23], controlled-release carriers $[24,25]$ or component in hydrogels [26]. XG is demonstrated to be biodegradable [27] and bioadhesive, which increases retention at the site of application [28,29]. Moreover, it also has the antineoplastic effects [30] and can promote wound-healing [31].

\section{APPLICATIONS OF XG ON OA TREATMENT}

\subsection{Preparation of XG Injection}

A high quality is necessary for $\mathrm{XG}$ injection including high viscosity, high purity and endotoxin-free. However, the purification of XG is difficult. XG fermented broth contains many undissolved matter such as bacterial cell residues, proteins and other biopolymers [32]. Furthermore, the purification processes need to not only obtain a high quality product but also suit for large-scale production. The high viscosity of XG fermented broth make the purification more difficult. To obtain high quality of XG for IA injection, the purification process containing the preparation and resolution of raw XG samples, $1 \%(\mathrm{w} / \mathrm{v})$ diatomite absorption, cake filtration, enzymolysis, $1 \%$ (w/v) active carbon absorption, cake filtration, fine filtration with $0.45 \mu \mathrm{m}$ microporous membrane, precipitation using isopropanol and stoving was established [17]. This purification process is easy to meet large-scale production, and the XG preparation obtained is high viscous.

\subsection{Safety Evaluation of XG Injection}

The safety of IA injection of XG was evaluated by Han, et al. [33]. When intra-articularly injected with $0.5 \%$ $2 \% \mathrm{XG}(0.1 \mathrm{~mL} / \mathrm{kg})$ in rabbit knee joint once a week for 5 weeks, there was no significant changes of the width of the knee joint, hematological and biochemical parameters observed, and no obvious histopathological changes in liver, kidney and knee joint detected [33]. In vitro experiments, the XG $(10-2000 \mu \mathrm{g} / \mathrm{mL})$ did not significantly modify chondrocytes viability and the production of matrix metalloproteinases (MMP), tissue inhibitor of metalloproteinase-1 (TIMP-1) in chondrocytes. XG displayed no cytotoxicity to rabbit chondrocytes [34]. However, the long-term toxicity of XG IA injection has not been evaluated.

\subsection{Therapy Effect of XG Injection on OA}

IA injection of XG may inhibit the continuous lesions on the cartilage and delay the progression of OA [17]. In an experimental model of OA induced by papain, IA injecttion of XG once every 2 weeks for 5 weeks decreased the severity of swelling of the knee joint, reduced the damage of cartilage surfaces, inhibited the chondrocytes changes, structural changes and loss of Safrain O staining intensity of femoral condyle and tibial plateau and also inhibited cells hyperplasia and infiltration of mononuclear cells in the synovium [17]. Futhermore, IA injection of XG once every 2 weeks for 5 weeks and IA injection of SH once a week for 5 weeks had similar effectiveness. IA injection of XG is likely to be injected fewer times than $\mathrm{SH}$ to get the same treatment results. XG could also preserve the proteoglycans (PG), which is the extracellular matrix mainly composed of. PGs maintain the water of articular cartilage and provide important biomechanical functions, such as load bearing, load distribution and shock absorption. When XG was added to interleukin-1 beta (IL-1 $\beta$ )-treated chondrocytes, there were significant restorations in chondrocytes prolifera- 
tion, inhibition in the protein expression of MMP-1, -3, and -13 , increase in TIMP-1 production in a dose-dependent manner in doses ranging from 100 to $1000 \mu \mathrm{g} / \mathrm{mL}$ [34].

\section{POSSIBLE ACTION MACHANISM OF XG INJECTION}

\subsection{Restore the Viscoelasticity of Synovium Fluid}

$\mathrm{OA}$ is a degenerative disease that appears to be caused by both biomechanical and biochemical factors [35]. Extensive investigations of femorotibial alignment have demonstrated that the mechanical forces play an important role in knee OA progression. Compared with normal synovial fluid, the synovial fluid in OA patients is markedly less viscoelastic due to lower concentration and reduced molecular weight of the SH, so its ability to lubricate and absorb shock is decreased [10]. Attention to the important role of mechanical factors in OA etiopathogenesis and therapies directed at unloading or reduceing the forces in the OA joints is required.

The high viscoelasticity of $\mathrm{XG}$ injection is crucial for the augmenting and elevating the viscoelastic properties of synovial fluid. XG preparations could behave as a viscous liquid at low shear rates and as an elastic solid at high shear rates. So XG could act as an elastic shock absorber during low impact movements of the joint and as a viscous lubricant during high impact movement. IA injection of XG may increase the ability of synovial fluid to lubricate and protect articular tissues, and to absorb joint loads, inhibiting the further progression of OA.

\subsection{Biochemical Actions}

Significant progress on the role of inflammation in OA has been made in recent years. Studies show that the excessive production of cytokines, inflammatory mediators and growth factors by the inflamed synovium and activated chondrocytes play a pivotal role in the pathophysiology of OA [36-38]. Osteoarthritic chondrocytes and the synovium from OA patients produce a number of inflammatory mediators including IL- $1 \beta$, tumor necrosis factor-alpha (TNF- $\alpha$ ), prostaglandins and nitric oxide (NO) [36,37]. Interleukins (ILs) are a family of interrelated cytokines that regulate the acute inflammation process. IL-1 $\beta$, a cytokine released by synovial cells and macrophages, plays a decisive role in amplifying inflammation in OA [39]. IL-1 $\beta$ can stimulate its own production and induce chondrocytes and synovial cells to produce other cytokines such as IL-8, IL-6, as well as stimulate proteases, $\mathrm{NO}$ and prostaglandin $\mathrm{E}_{2}\left(\mathrm{PG} \mathrm{E}_{2}\right)$ production. IL-1 $\beta$ can alter chondrocytes anabolism by enhancing PG degradation and repressing the synthesis of PG. IL-1 $\beta$ up-regulates matrix degrading enzymes such as MMPs and down-regulates the TIMPs in chondrocytes [40]. Excess of MMPs plays an important role in cartilage breakdown, because of their ability to degrade the extracellular matrix proteins. The imbalance between MMPs and TIMPs is of great importance in the progression of OA [41].

XG has been demonstrated to have some biochemical actions on chondrocytes in vitro. Previous study showed that when XG was added to IL- $1 \beta$-treated chondrocytes, there were significant restorations in chondrocytes proliferation, inhibition in the protein expression of MMP-1, -3 , and -13 , increase in TIMP-1 production. It was speculated that the protective effect of $\mathrm{XG}$ on cartilage might be associated with the prevention of destruction of IL- $1 \beta$ on chondrocytes and the regulation of MMP- $1,-3$, -13 , TIMP-1 [33].

\section{FUTURE PROSPECT}

IA injection of XG is a potential therapy for OA. However, there are still many works to do for the development of this new treatment method. The future study will be designed to solve the following questions.

1) Whether $X G$ could relieve OA pain

Pain is one of the most important symptoms of OA. The presence of local joint inflammation, altered cartilage and bone turnover in OA can lead to joint pain. The activation and release of local pro-inflammatory mediators in the joint such as prostaglandins and cytokines influence excitability of nociceptors of articular nerves [38]. However, angiogenesis in OA promotes ingrowth of new sensory nerves into peripheral joint tissue exposed to inflammation and damage, which contributes to persistent pain [42]. In experimental OA, articular nerves become hyperalgesic, spontaneously discharge, and are sensitive to non-noxious joint movements [38].

IA injection of XG with high viscosity is considered to be able to coat the receptor endings of the joint pain fibers, decrease their sensitization to inflammatory mediators, entrapment of endogenous pain substances or decreased activation to their receptor. IA injection of $X G$ is very likely to relieve the OA pain. Our studies of XG on body torsion experiment in mice and the capillary permeability experiment showed that intraperitoneal injecttion of XG could reduce the times of torsion and inhibit capillary permeability (results not be published), supporting the hypothesis above. However, to prove the hypothesis that IA injection of XG may relieve OA pain, future study will be conducted.

2) Detailed action mechanism of $X G$

The OA pathogenesis is complex and the detailed mechanism of XG in chondrocytes and the inflammatory is not clear. Further study is needed to understand the detailed action mechanism of XG injection. For example, whether XG could affect the NO expressed in OA joint. 
In OA chondrocytes, the inducible nitric oxide synthase (iNOS) enzyme is unregulated, resulting in over expression of NO and perpetuating the release of inflammatory cytokines and other catabolic processes. NO promotes chondrocyte inflammatory responses, mediates chondrocyte apoptosis, inhibits both PG and collagen synthesis, and activates MMPs. All of these activities contribute to the catabolic consequences of $\mathrm{NO}$ in cartilage. In vivo and in vitro experiments are necessary to know whether $\mathrm{XG}$ injection can be able to prevent the production of $\mathrm{NO}$ in OA [35].

3) Pharmacokinetic of $X G$ injection

The efficacy of XG on OA seems to be highly related to its residence time in the joint cavity after injection. The actual joint distribution, residence time, mechanism of removal and degradation of the XG injection will be detailed studied.

4) Longer therapy effect of $X G$ on $O A$

To evaluate the long-lasting effect of $\mathrm{XG}$ injection, the anterior cruciate ligament transection (ACLT) OA model or other experiment OA model will be adopted with the observation period extended.

\section{ACKNOWLEDGEMENTS}

This work was supported by major projects of independent innovation achievements (No. 2009ZHZXIC1001) grant from Science and Technology Department of Shandong Province, China.

\section{REFERENCES}

[1] Woolf, A.D. and Pfleger, B. (2003) Burden of major musculoskeletal conditions. Bull World Health Organ, 81, 646-656.

[2] Scanzello, C.R. and Goldring, S.R. (2012) The role of synovitis in osteoarthritis pathogenesis. Bone, 51, 249257. doi:10.1016/j.bone.2012.02.012

[3] Sharma, L., Kapoor, D. and Issa, S. (2006) Epidemiology of osteoarthritis: An update. Current Opinion in Rheumatology, 18, 147-156. doi:10.1097/01.bor.0000209426.84775.f8

[4] Felson, D.T., et al. (1988) Obesity and knee osteoarthritis. The Framingham study. Annals of Internal Medicine, 109, 18-24.

[5] American College of Rheumatology Subcommittee on Osteoarthritis Guidelines (2000) Recommendations for the medial management of osteoarthritis of the hip and knee. Arthritis \& Rheumatism-Aarthritis Care \& Research, 43, 1905-1915.

[6] Jackson, D.W. and Simon, T.M. (2006) Intra-articular distribution and residence time of Hylan A and B: A study in the goat knee. Osteoarthritis and Cartilage, 14, 12481257. doi: 10.1016/j.joca.2006.05.015

[7] Boettger, M.K., et al. (2011) Evaluation of long-term antinociceptive properties of stabilized hyaluronic acid preparation (NASHA) in an animal model of repetitive joint pain. Arthritis Research and Therapy, 13, R110. doi:10.1186/ar3394

[8] Mihara, M., et al. (2007) Different effects of high molecular weight sodium hyaluronate and NSAID on the progression of the cartilage degeneration in rabbit OA model. Osteoarthritis and Cartilage, 15, 543-549. doi:10.1016/j.joca.2006.11.001

[9] Bannuru, R.R., et al. (2011) Therapeutic trajectory following intra-articular hyaluronic acid injection in knee osteoarthritis-meta-analysis. Osteoarthritis and Cartilage, 19, 611-619. doi:10.1016/j.joca.2010.09.014

[10] Balazs, E. (1982) The physical properties of synovial fluid and the specific role of hyaluronic acid. In: Helfet, A.J., Ed., Disorders of the Knee, JB Lippincott, Philadelphia, 61-74.

[11] Puh, W., et al. (1993) Intra-articular sodium hyaluronate in osteoarthritis of the knee: A multicenter, doubleblinded study. Osteoarthritis and Cartilage, 1, 233-241. doi:10.1016/S1063-4584(05)80329-2

[12] Gomis, A., et al. (2009) Intra-articular injections of hyaluronan solutions of different elastoviscosity reduce nociceptive nerve activity in a model of osteoarthritic knee joint of the guinea pig. Osteoarthritis Cartilage, 17, 798804. doi:10.1016/j.joca.2008.11.013

[13] Barbucci, R., et al. (2002) Hyaluronic acid hydrogel in the treatment of osteoarthritis. Biomaterials, 23, 45034513. doi:10.1016/S0142-9612(02)00194-1

[14] García-Ochoa, F., et al. (2000) Xanthan gum: Production, recovery, and properties. Biotechnology Advances, 18, 549-579. doi:10.1016/S0734-9750(00)00050-1

[15] García-Abuín, A., et al. (2011) Viscosimetric behaviour of hyaluronic acid in different aqueous solutions. Carbohydrate Polymers, 85, 500-505. doi:10.1016/j.carbpol.2011.02.028

[16] Bewersdorff, H.W. and Singh, R.P. (1988) Rheological and drag reduction characteristics of xanthan gum solutions. Rheologica Acta, 27, 617-627. doi:10.1007/BF01337457

[17] Han, G.Y., et al. (2012) Preparation of xanthan gum injection and its protective effect on articular cartilage in the development of osteoarthritis. Carbohydrate Polymers, 87, 1837-1842. doi:10.1016/j.carbpol.2011.10.016

[18] Cadmus, M.C., et al. (1976) Colonial variation in Xanthomonas campestris NRRL B-1459 and characterization of the polysaccharide from a variant strain. Canadian Journal of Microbiology, 22, 942-948. doi: $10.1139 / \mathrm{m} 76-136$

[19] Jansson, P.E., Kenne, L. and Lindberg, B. (1975) Structure of the extracellular polysaccharide from Xanthomonas campestris. Carbohydrate Research, 45, 275-282. doi:10.1016/S0008-6215(00)85885-1

[20] Kennedy, J.F. and Bradshaw, I.J. (1984) Production, properties and applications of xanthan. Progress in Industrial Microbiology, 19, 319-371.

[21] Ceulemans, J., Vinckier, I. and Ludwig, A. (2002) The use of xanthan gum in an ophthalmic liquid dosage form: rheological characterization of the interaction with mucin. Journal of Pharmaceutical Sciences, 1, 1117-1127. 


\section{doi:10.1002/jps.10106}

[22] Harward, I., Schenker, M.D. and Lewis, H. (2000) Longterm intraocular pressure-lowering efficacy and safety of timolol maleate. American Journal of Ophthalmology, 130, 145-150.

[23] Baroud, G., et al. (2004) Injection biomechanics of bone cements used in vertebroplasty. Bio-Medical Materials and Engineering, 14, 487-504.

[24] Talukdar, M.M., et al. (1996) Comparative study on xanthan gum and hydroxypropylmethyl cellulose as matrices for controlled-release drug delivery I. Compaction and in vitro drug release behaviour. International Journal of Pharmaceutics, 129, 233-241. doi:10.1016/0378-5173(95)04355-1

[25] Mikac, U., et al. (2010) A new approach combining different MRI methods to provide detailed view on swelling dynamics of xanthan tablets influencing drug release at different $\mathrm{pH}$ and ionic strength. Journal of Controlled Release, 145, 247-256. doi:10.1016/j.jconrel.2010.04.018

[26] Chen, H.B., et al. (2006) Microemulsion-based hydrogel ormulation of ibuprofen for topical delivery. Journal of Biomedical Materials Research, 315, 52-58.

[27] Chellat, F., et al. (2000) Study of biodegradation behavior of chitosan-xanthan microspheres in simulated physiological media. Journal of Biomedical Materials Research, 53, 592-599.

doi:10.1002/1097-4636(200009)53:5<592::AID-JBM20> 3.0.CO;2-P

[28] Nakamura, F., et al. (1996) In vitro and in vivo nasal mucoadhesion of some water-soluble polymers. International Journal of Pharmaceutics, 134, 173-181. doi:10.1016/0378-5173(95)04416-7

[29] Chiou, C.J., Tseng, L. P. and Deng, M.C. (2009) Mucoadhesive liposomes for intranasal immunization with an avian influenza virus vaccine in chickens. Biomaterials, 30, 5862-5868. doi:10.1016/j.biomaterials.2009.06.046

[30] Takeuchi, A., et al. (2009) Oral administration of xanthan gum enhances antitumor activity through Toll-like receptor 4. International Immunopharmacology, 9, 1562-1567. doi:10.1016/i.intimp.2009.09.012

[31] Matthews, K.H., et al. (2005) Lyophilised wafers as a drug delivery system for wound healing containing methylcellulose as a viscosity modifier. International Journal of Pharmaceutics, 289, 51-62. doi:10.1016/j.ijpharm.2004.10.022

[32] Salah, R.B., et al. (2010) Optimisation of xanthan gum production by palm date (Phoenix dactylifera L.) juice by-products using response surface methodology. Food Chemistry, 121, 627-633. doi:10.1016/j.foodchem.2009.12.077

[33] Han, G.Y., et al. (2012) Safety evaluation of intra- articular injection of xanthan gum into knee joint of rabbit. Chinese Journal of Biochemical Pharmaceuticals, 33 109-111.

[34] Han, G.Y., et al. (2012) The protective effect of xanthan gum on interleukin-1 $\beta$ induced rabbit chondrocytes. Carbohydrate Polymers, (in Press). doi:10.1016/j.carbpol.2012.04.023

[35] Steven, B. and Abramson, M.D. (2008) Osteoarthritis and nitric oxide. Osteoarthritis and Cartilage, 16, 15-20. doi:10.1016/S1063-4584(08)60008-4

[36] Goldring, M.B. and Goldring, S.R. (2007) Osteoarthritis. Journal of Physiology-Cell Physiology, 213, 626-634. doi: $10.1002 /$ jcp. 21258

[37] Bonnet, C.S. and Walsh, D.A. (2005) Osteoarthritis, angiogenesis and inflammation. Rheumatology, 44, 7-16. doi:10.1093/rheumatology/keh344

[38] Sofat, N., Ejindu, V. and Kiely, P. (2011) What makes osteoarthritis painful? The evidence for local and central pain processing. Rheumatology, 50, 2157-2165. doi:10.1093/rheumatology/ker283

[39] Chowdhury, T.T., Bader, D.L. and Lee, D.A. (2006) Antiinflammatory effects of IL-4 and dynamic compression in IL-1beta stimulated chondrocytes. Biochemical and Biophysical Research Communications, 339, 241-247. doi:10.1016/j.bbrc.2005.11.016

[40] Aida, Y., et al. (2005) The effect of IL-1beta on the expression of matrix metalloproteinases and tissue inhibitors of matrix metalloproteinases in human chondrocytes. Life Sciences, 77, 3210-3221. doi:10.1016/j.lfs.2005.05.052

[41] Burrage, P.S., Mix, K.S. and Brinckerhoff, C.E. (2006) Matrix metalloproteinases: Role in arthritis, Front. Bioscience, 11, 529-543. doi:10.2741/1817

[42] McDougall, J.J., Watkins, L. and Li, Z. (2006) Vasoactive intestinal peptide (VIP) is a modulator of joint pain in a rat model of osteoarthritis. Pain, 123, 98-105. doi:10.1016/j.pain.2006.02.015 